\title{
Structural Health Monitoring of Composite Plates Under Ambient and Cryogenic Conditions
}

\author{
Robert C. Engberg* \\ NASA Marshall Space Flight Center, Huntsville, AL, 35812
}

\begin{abstract}
Methods for structural health monitoring are now being assessed, especially in high-performance, extreme environment, safety-critical applications. One such application is for composite cryogenic fuel tanks. The work presented here attempts to characterize and investigate the feasibility of using imbedded piezoelectric sensors to detect cracks and delaminations under cryogenic and ambient conditions. Different types of excitation and response signals and different sensors are employed in composite plate samples to aid in determining an optimal algorithm, sensor placement strategy, and type of imbedded sensor to use. Variations of frequency and high frequency chirps of the sensors are employed and compared. Statistical and analytic techniques are then used to determine which method is most desirable for a specific type of damage and operating environment. These results are furthermore compared with previous work using externally mounted sensors. More work is needed to accurately account for changes in temperature seen in these environments and be statistically significant. Sensor development and placement strategy are other areas of further work to make structural health monitoring more robust. Results from this and other work might then be incorporated into a larger composite structure to validate and assess its structural health. This could prove to be important in the development and qualification of any $2^{\text {nd }}$ generation reusable launch vehicle using composites as a structural element.
\end{abstract}

\section{Nomenclature}

$a=$ geometric constant of piezoelectric material

$\alpha=$ coefficient of thermal expansion

$\boldsymbol{d}_{z j} \quad=$ piezoelectric coupling tensor

$\delta=\quad$ dielectric loss tangent to the piezoelectric sensor

$E \quad=$ Young's modulus

$\varepsilon \quad=$ strain

$\varepsilon_{z z} \quad=$ strain tensor

$i \quad=$ complex number

$v \quad=$ Poisson's ratio

$\omega \quad=$ natural frequency

$T \quad=$ temperature

$Y \quad=$ electrical admittance

$Z \quad=$ mechanical impedance

\section{Introduction}

A $\mathrm{S}$ composites become more widespread in high performance structures due to their high strength-to-weight properties and ability to be molded to a desired shape, accurate, reliable, minimally intrusive, and affordable non-destructive evaluation of these materials often remains an elusive task. X-ray and C-scan equipment are good for detecting structural and material faults in a laboratory environment, but generally are impractical for larger and

\footnotetext{
* Aerospace Test Engineer, Structural Dynamics Test Branch, Mail Stop ET23, MSFC, AL 35812. AIAA Member.
} 
more complex structures. Modal and acoustic testing and analysis are another alternative, but their complexity and sensitivity to changing boundary conditions make them impractical. Visual inspection is often subjective and generally unable to detect microcracking. From this and other related studies, it is hoped that using a network of imbedded piezoelectric sensors exciting the structure at high frequencies can lead to a reliable, robust, and accurate structural health monitoring (SHM) system.

Part of the motivation for this work stems from the now cancelled X-33 liquid hydrogen composite tank failure and the desire to ensure such a failure does not recur with any $2^{\text {nd }}$ generation reusable launch vehicle. The failure investigation team at NASA concluded that the most prohable cause of the X-33 tank failure was a combination of several factors, including microcracking of the inner facesheet, degraded bonding of the internal honeycomb core, and the presence of defects, damage, and untested flaws within the tank. The net result of these flaws was that liquid hydrogen propellant was allowed to permeate through the facesheeet and into the honeycomb area, boiling and expanding into gas, and rupturing the tank once the propellant was discharged and the tank warmed to ambient temperatures.

This study was an attempt to investigate the feasibility of using high frequency, ultrasonic signals introduced into piezoelectric sensors to determine trends for piezoelectric material selection, mounting of sensors, frequency ranges, and ideal placement strategy for structural health monitoring of cryogenic vessels. Both impedance and pulse Lamb wave sensor interrogation methods were compared to help characterize how imbedded sensors respond and react in cryogenic environments.

\section{Analytical Background}

For this study, two common types of piezoelectric material were used: a lead-zirconate-titanium alloy $\mathrm{Pb}\left(\mathrm{Zr}_{1-\mathrm{x}} \mathrm{Ti}_{\mathrm{x}}\right) \mathrm{O}_{3}$ (abbreviated PZT), and the single crystal piezoelectric ( $\mathrm{SCP}) \mathrm{Pb}\left(\mathrm{Mg}_{1-3} \mathrm{Nb}_{2-3}\right) \mathrm{O}_{3}-\mathrm{PbTiO}_{3}$ (abbreviated PMN-PT). The PZT, made by Piezo Systems, Inc., came in 3-inch square sheets that were subsequently cut into pieces to fit in the test material. The SCP's, made by TRS Ceramics, were $1 \mathrm{~cm}$ in diameter. Advantages to using PZT are low cost, ability to cut down to a desired shape, and overall high piezoelectric activity in all directions. Advantages to using SCP are higher piezoelectric coefficients than PZT and higher frequency response.

For impedance measurements of the sensors, high frequency sinusoidal sweeps were inputted into each sensor while the resulting real and complex measurements of the piezoelectric effect were measured. Since the sensor is either bonded to or imbedded within the structure, the structure in turn is then deformed along with the sensor once a drive signal is sent to the sensor to produce a localized vibration. The coupled electromechanical admittance (inverse of impedance) can be expressed as ${ }^{3}$

$$
Y=\operatorname{Re}(Y)+i \operatorname{Im}(Y)
$$

The solution to the wave equation for electrical admittance can be expressed as ${ }^{2}$

$$
Y(\omega)=i \omega a\left[\boldsymbol{\varepsilon}_{\mathrm{zz}}(1-i \delta)-\frac{Z_{s}(\omega)}{Z_{s}(\omega)+Z_{a}(\omega)} \boldsymbol{d}_{z j}{ }^{2} E_{p_{j}}\right]
$$

where $\mathrm{Y}$ is the admittance, $\omega$ is the natural frequency, $a$ is the geometric constant of the piezoelectric material, $\varepsilon_{z z}$ is the strain tensor, $\delta$ is the dielectric loss tangent to the piezoelectric sensor, $Z_{a}$ and $Z_{s}$ are the sensor's and structure's mechanical impedances, respectively, $\boldsymbol{d}_{z j}$ is the piezoelectric coupling tensor, and $E_{p}$ is the Young's modulus of the piezoelectric material.

Since the imbedded sensors in the material have different coefficients of thermal expansion (CTE), thermal stresses and strains will develop when undergoing a large temperature change. Part of the reason for the baseline thermal testing at cryogenic temperatures $\left(-400^{\circ} \mathrm{F}\right)$ was to validate the ability of the sensors to detect these thermal strains

To analyze the thermal stresses and strains, the thermal strain can be expressed as

$$
\varepsilon=\alpha \Delta T \text {. }
$$

2

American Institute of Aeronautics and Astronautics 
where $\alpha$ is the coefficient of thermal expansion and $\Delta T$ is the change in temperature. Expanding this into Cartesian coordinates and incorporating stresses yields

$$
\begin{aligned}
& \varepsilon_{x}=\frac{1}{E}\left[\sigma_{x}-v\left(\sigma_{y}+\sigma_{z}\right)\right]+\alpha \Delta T \\
& \varepsilon_{y}=\frac{1}{E}\left[\sigma_{y}-v\left(\sigma_{x}+\sigma_{z}\right)\right]+\alpha \Delta T \\
& \varepsilon_{z}=\frac{1}{E}\left[\sigma_{z}-v\left(\sigma_{x}+\sigma_{y}\right)\right]+\alpha \Delta T .
\end{aligned}
$$

or the specimen plates, they were not totally fixed at their edges, so the thermal stress inside the plate were expressed in a simplified form as ${ }^{4}$

$$
\sigma_{i}=\frac{-E_{i} \alpha \Delta T}{1-v}
$$

But the circular SCPs were assumed to be fixed inside the material, and therefore the stress equation used was

$$
\sigma_{i_{\max }}=\frac{E_{i} \alpha \Delta T}{2(1-v)}
$$

The square PZTs could then be expressed as

$$
\sigma_{i_{\max }}=\frac{E_{i} \alpha \Delta T}{2}
$$

\section{Test Description}

In the initial planning of the test specimen selection, both a unidirectional epoxy matrix pair and a carbon fiber epoxy matrix pair were to be fabricated, each with four piezoelectric sensors (two PZTs and two SCPs) imbedded in the corners of the material. However, time and cost constraints limited the production of just one pair of specimens. The material chosen was the unidirectional epoxy matrix composite, due to its good permeability properties, impact resistance, and high tensile strength that would make this material a good candidate for a cryogenic fuel tank. Small gauge wires were then placed on either side of the piezoelectric sensors and fastened with Kapton ${ }^{\mathrm{TM}}$ tape. The layout of the sensors can be seen in Figure 1. However, this material had a high prepreg temperature that resulted in some depoling of the piezos. Measurements made of the sensors themselves with a capacitor-inductor analyzer after prepreg indicated a loss of around $25-30 \% \mathrm{pF}$ of charge.

Another material specimen was constructed, this one a polymer enhanced, graphite reinforced cementitious composite (PEGRCC). This material was developed at the University of Alabama in Huntsville (UAH) to demonstrate how it could be used in a variety of demanding applications, since it possesses the high compressive strength of cement and the high tensile strength of carbon fiber graphite mesh while being relatively light weight and low cost. Such a material was recently used for the fuselage of an experimental rocket fired by UAH engineering students to prove its effectiveness and suitability for aerospace applications. 


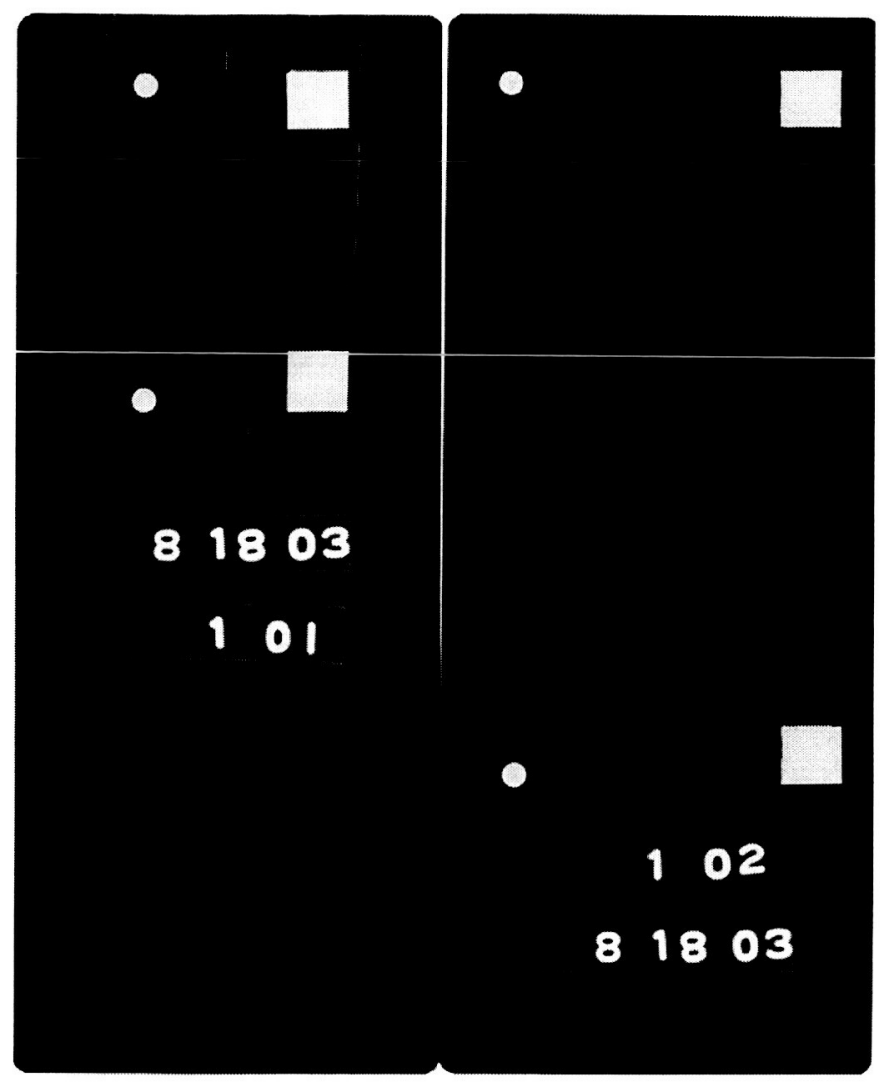

Figure 1: X-ray images of unitape epoxy composite materials with imbedded sensors. The square sensors are PZT patches; the round sensors are SCP patches.

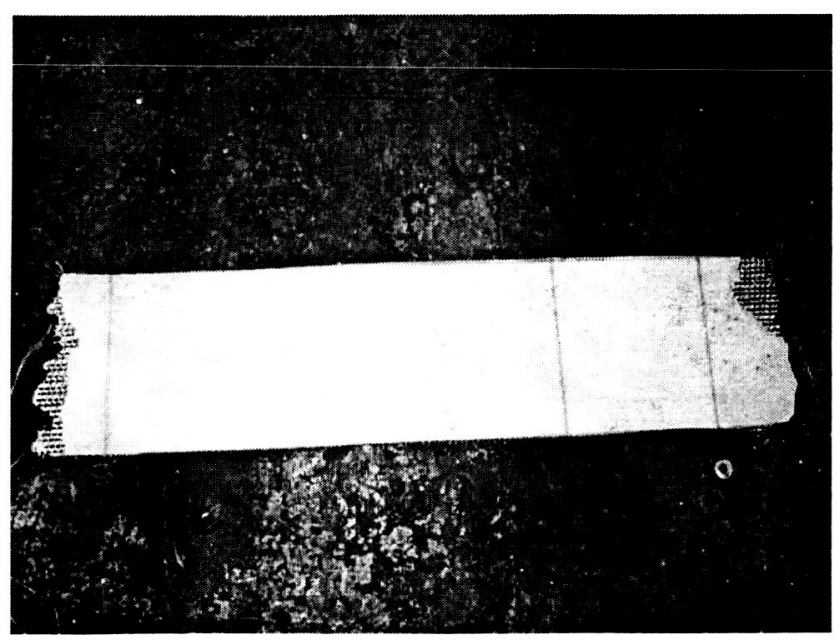

Figure 2: PEGRCC test specimen with imbedded sensors. 
Each specimen was to have two PZT sensors in the corners on one side and two SCP sensors on the other. This was to test the sensors' ability to detect induced damage in the center of the specimen. Figure 1 shows X-ray images of the two unitape composite specimens used in this work. Lamb waves were also used in such a way that both types of sensor material were emitter or receiver of ultrasonic signals throughout the material. Post-processing of the timedomain signals involved conversion of the receiving signals of the sensor to frequency domain using an FFT transform and subsequent power spectral density.

Both sensors were subjected to the same type of cryogenic environment and material application. However, the thieminal shiock effccts from sudden exposure to cryogenic fluids were also a matter of investigation Therefore, hoth a gradual cryopumping of the materials in a cryogenic vacuum chamber and a sudden immersion into liquid nitrogen (LN2) were compared. For the unidirectional graphite epoxy matrix with imbedded piezoelectric sensors, little difference was found between emersion in liquid nitrogen and gradual reduction in temperature in the cryogenic vacuum chamber. The PEGRCC specimen, however, showed some detrimental effects of direct immersion into a cryogenic liquid, possibly due to penetration of the cryogenic liquid in between layers of the cut area of the concrete in which the sensors were exposed (Fig 9 and 10). Due to its porosity and high water content, the PEGRCC specimen could not be tested inside the cryogenic vacuum test chamber.

A variety of damage detection criteria exists when monitoring the real output of the impedance $Z_{p}$. Some involve monitoring statistical correlation over a given bandwidth; others involve monitoring changes in phase or magnitude of the measured impedance. The damage detection criterion chosen for this work uses a relatively simple damage metric that is the root mean square deviation (RMSD) of the impedance $Z_{p}$ taken before and after suspected damage 5

$$
\operatorname{RMSD}(\%)=\sqrt{\frac{\sum_{j=1}^{n}\left(Z_{p j}^{1}-Z_{p j}^{0}\right)^{2}}{\sum_{j=1}^{n}\left(Z_{p j}^{0}\right)^{2}}} \times 100 \%
$$

\section{Test Results and Conclusions}

At the minimum, the imbedded sensors appeared to be able to sense the cryogenic environment that they were in and showed some ability to detect cracking of the plate specimen. For the unidirectional epoxy matrix composite, all impedance sensors showed indications of thermal stresses as seen in the plots (Fig. 5 \& 6). They were different from the results of externally mounted sensors (Fig. $3 \& 4$ ) on other composites in that the impedance measurements were far more linear ${ }^{1}$. The externally mounted sensors were less constrained than the imbedded ones. Therefore, another possible application of the imbedded sensors could be a cryogenic fuel level avionics feedback sensor inside a tank, although further modeling and validation for repeatability must be done.

The relatively short length of the specimens appeared to limit the use of echoed impulses, so only impulses from an emitter sensor and the corresponding measurements of a receiver sensor were considered in this study. They appear to have some ability for crack detection, although a larger sample size of specimens is needed to be statistically confident. Little difference in measurements were seen in the longer and shorter length composite panels (see Fig $7 \& 8$ ).

Both the PZT and SCP type sensors appear to be suitable for use in cryogenic environments. Furthermore, both lower and higher frequency measurements appeared to have a good ability to detect damage. Figures 11 and 12 show that the composites have some ability to detect both damage and temperature effects, although a larger specimen sample size is necessary to be statistically significant. The effects of hydrogen embrittlement on a unitape composite material used in this study are a little-known-about phenomenon. Other tests could be done to assess operation at higher temperatures that do not exceed the Curie temperature to cause significant depoling. Further studies could also be done on imbedded sensors within a longer, narrower specimen to test a pulse-echo damage detection algorithm 


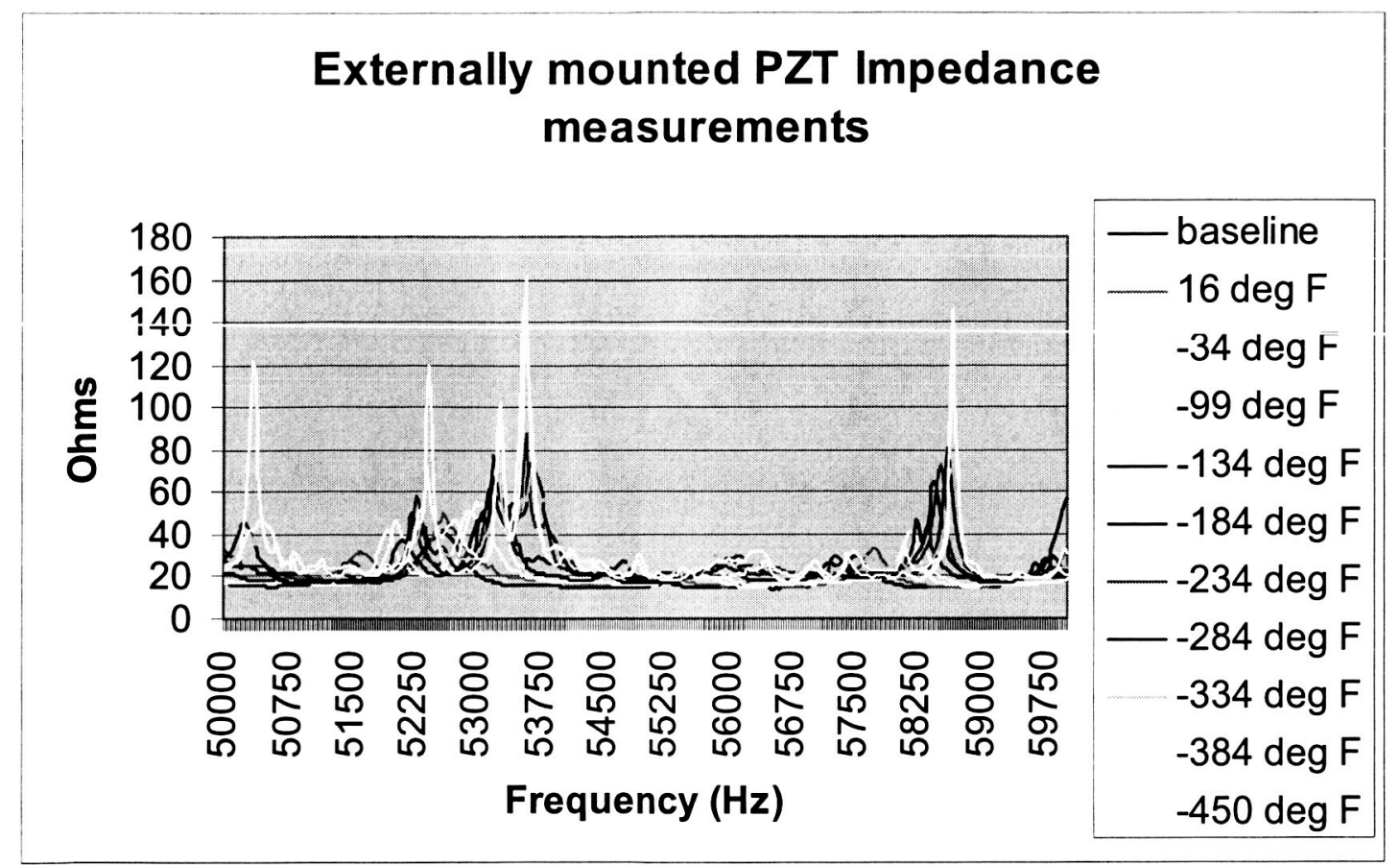

Figure 3

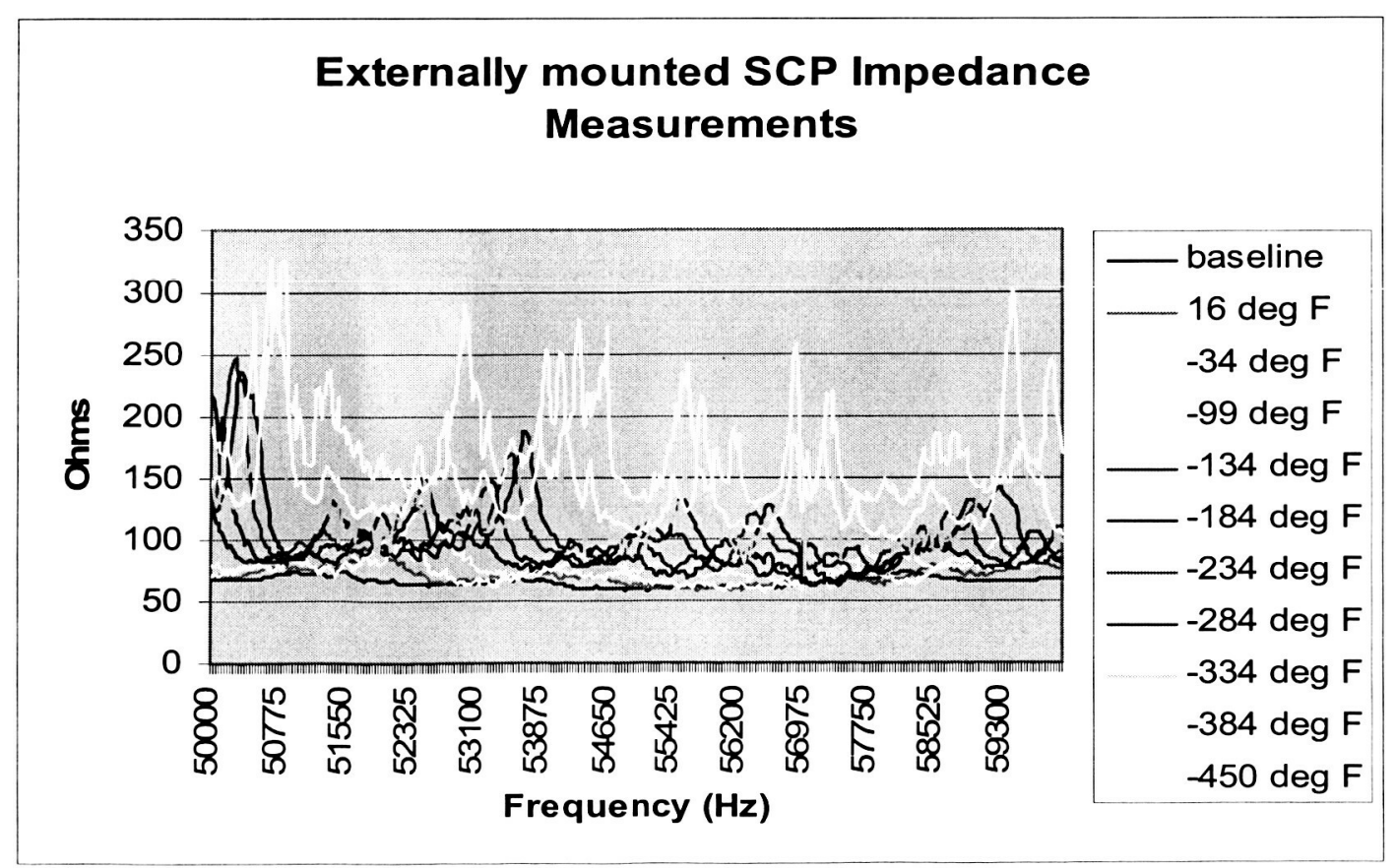

Figure 4 


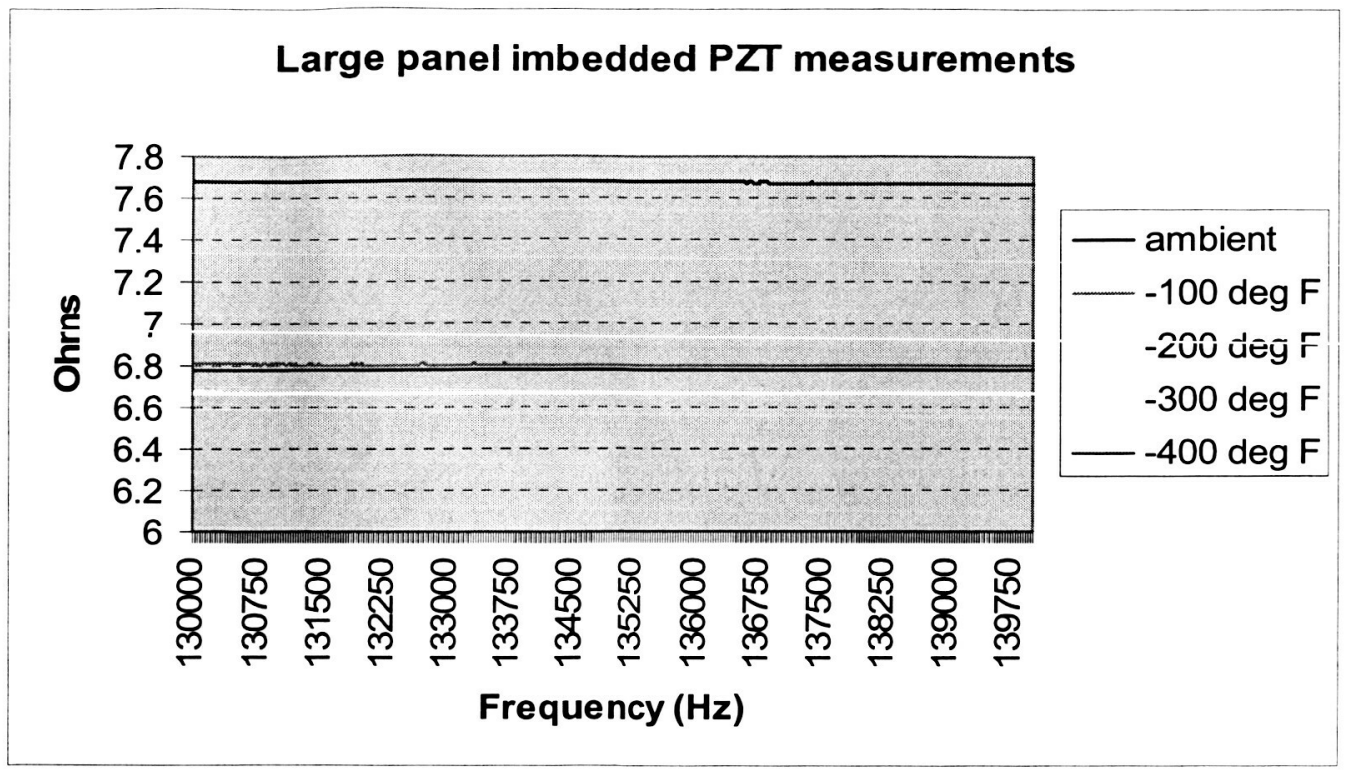

Figure 5

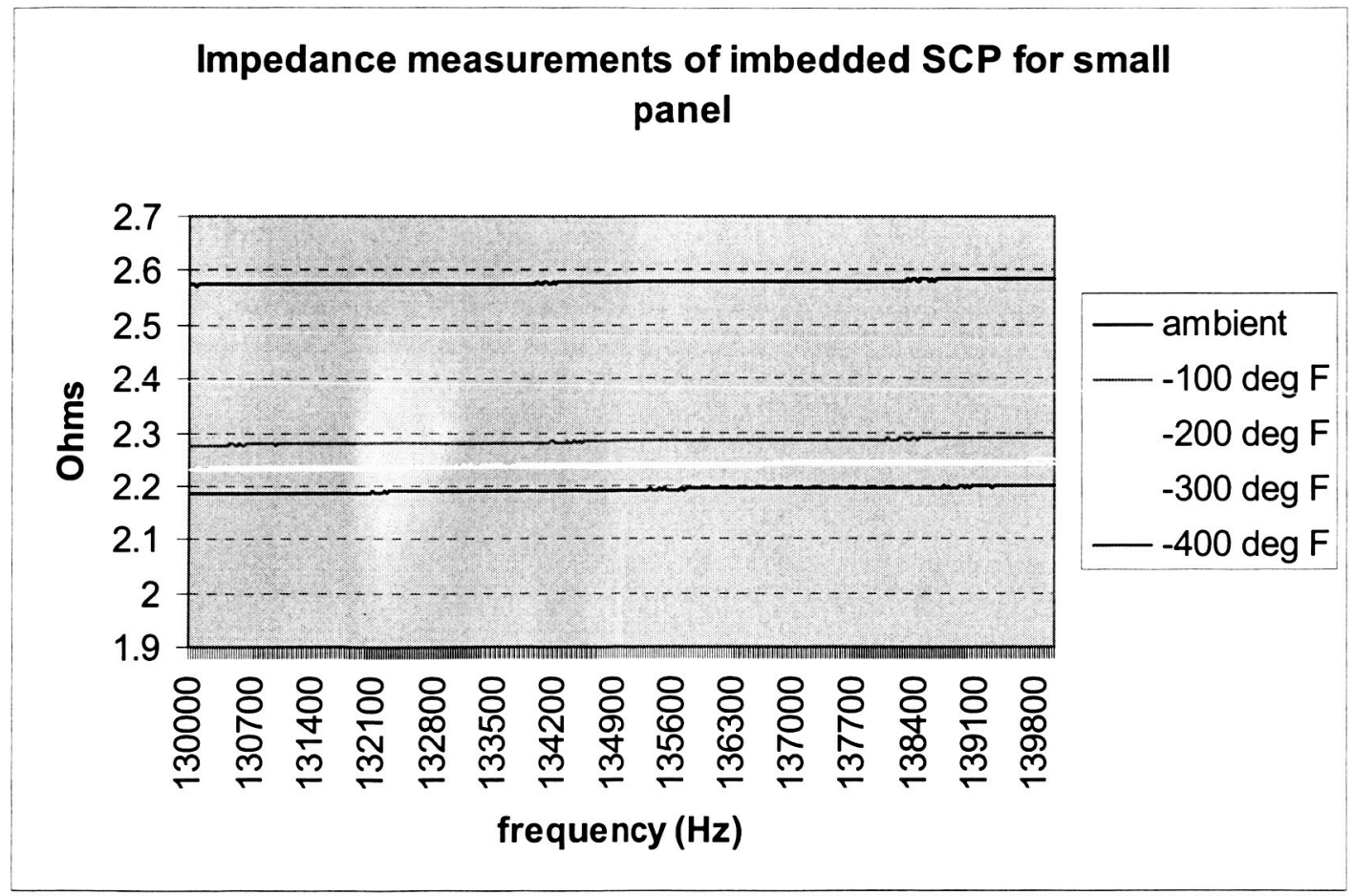

Figure 6 


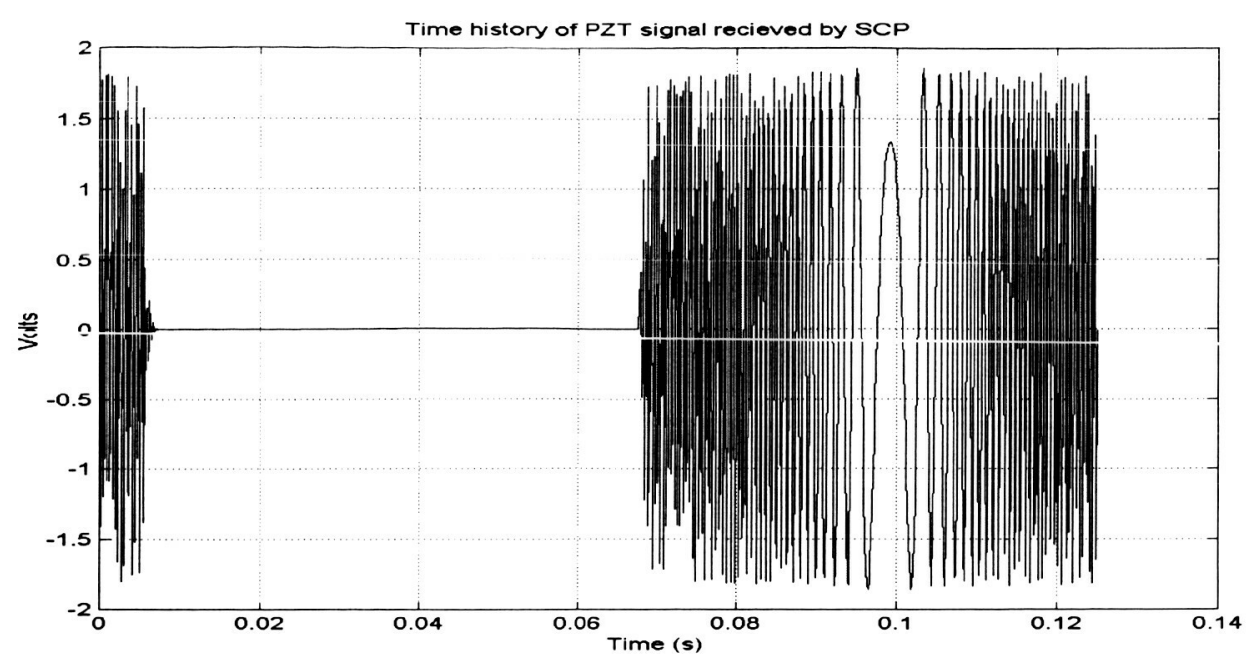

Figure 7

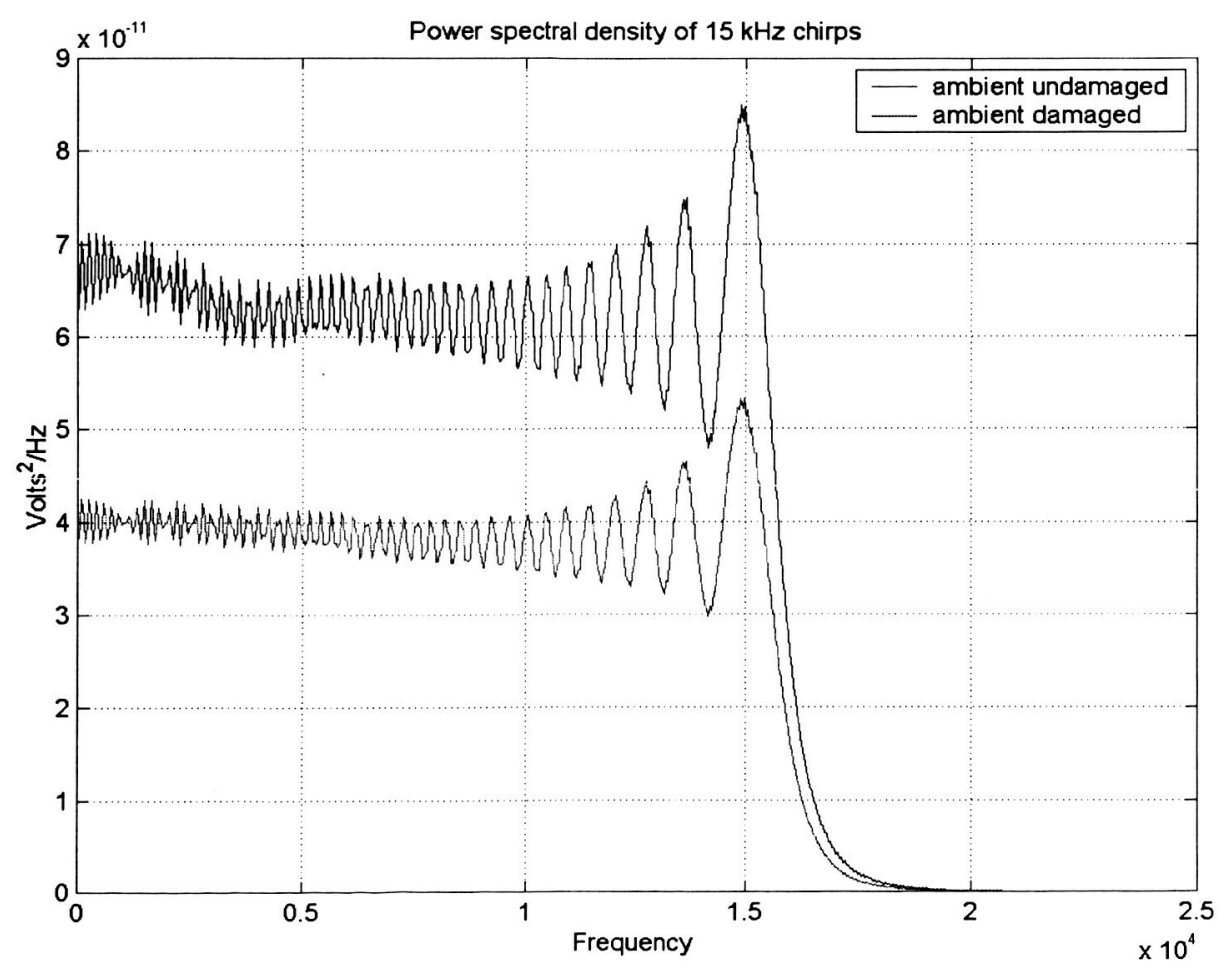

Figure 8 


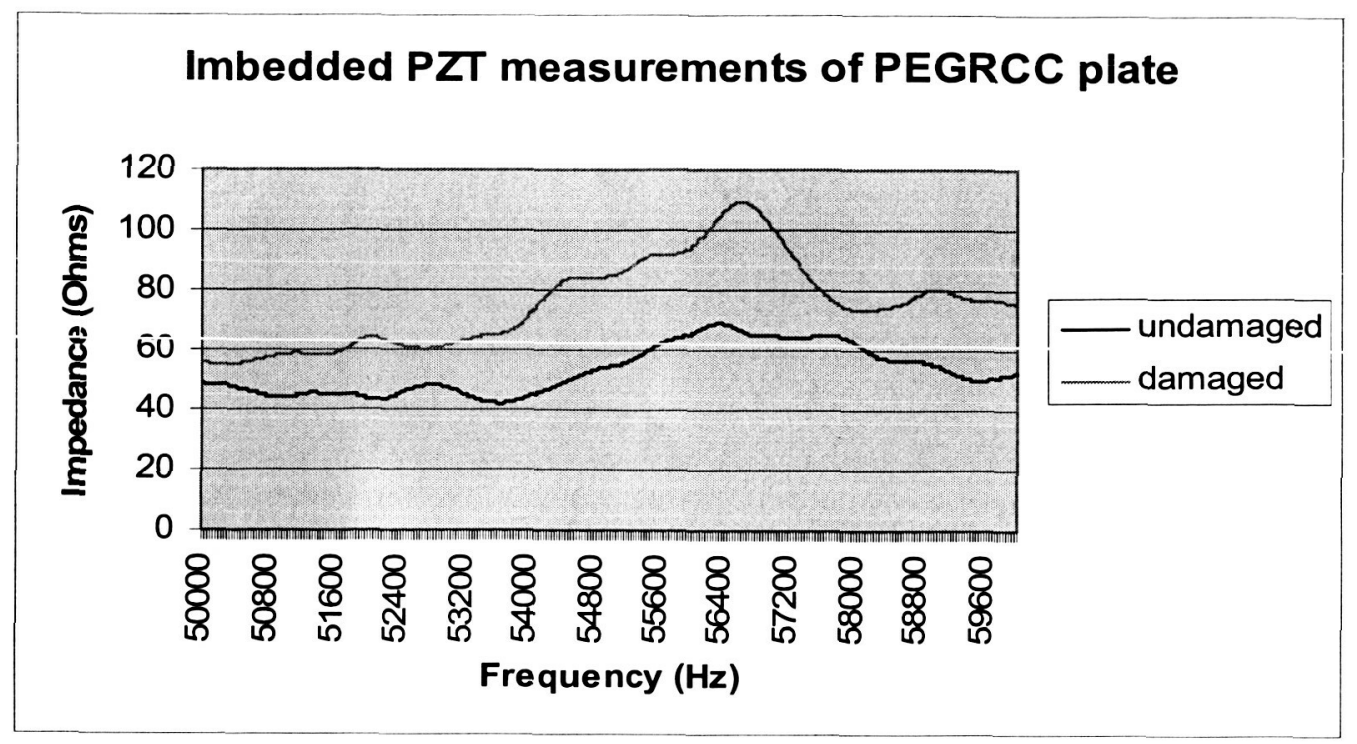

Figure 9

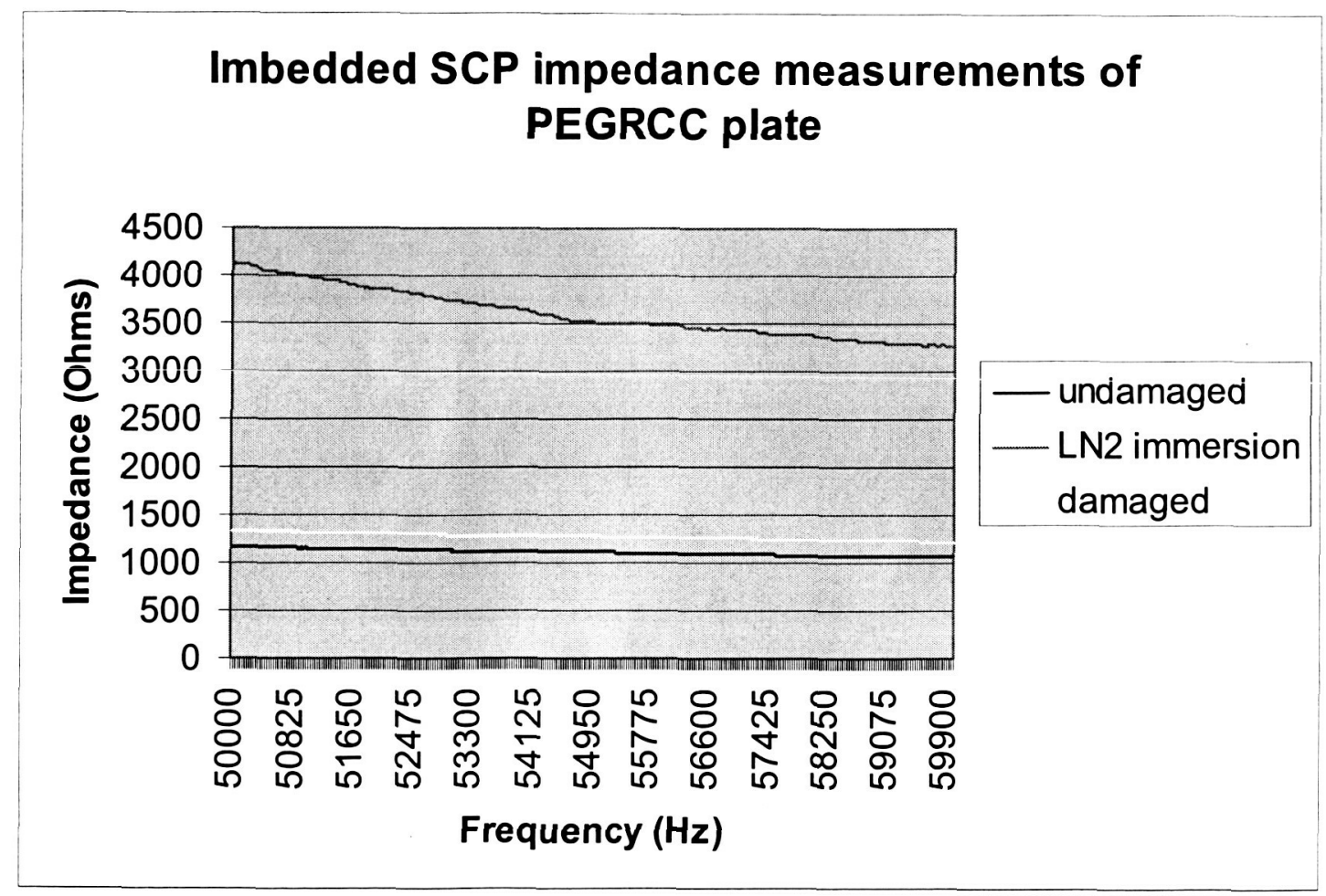

Figure 10 


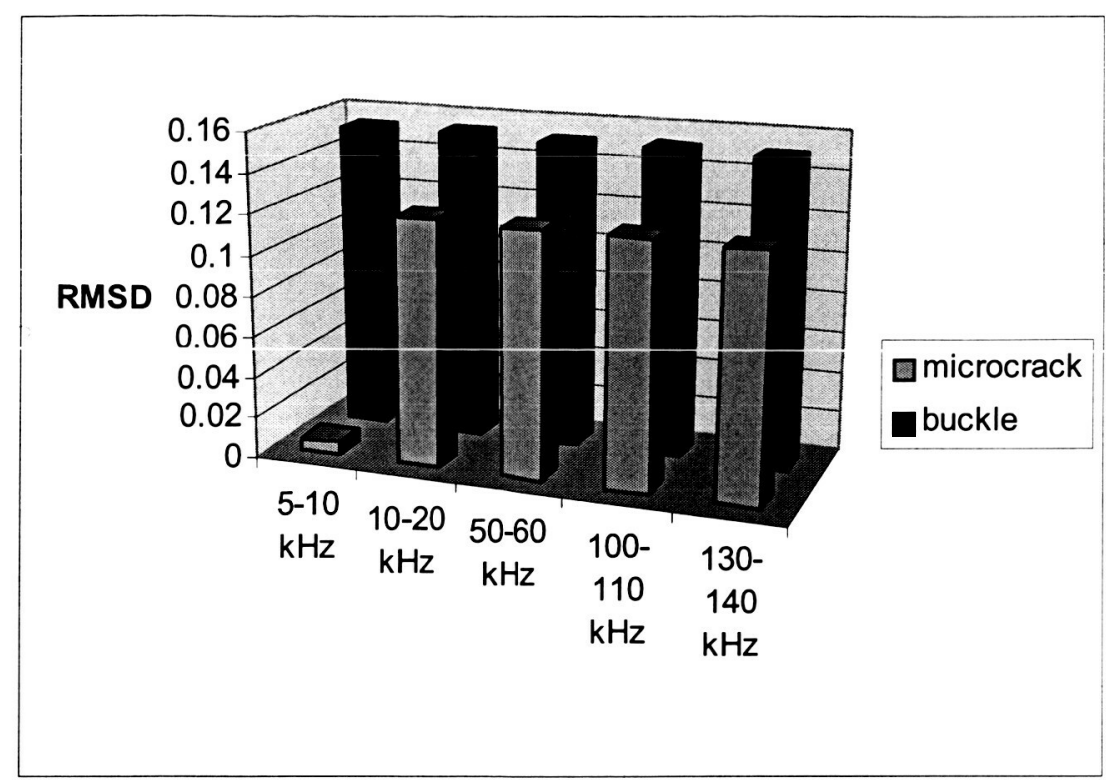

Figure 11: Root Mean Square Deviation (RMSD) of PZT impedance measurements of 4 x 6 panel after microcracking and buckling.

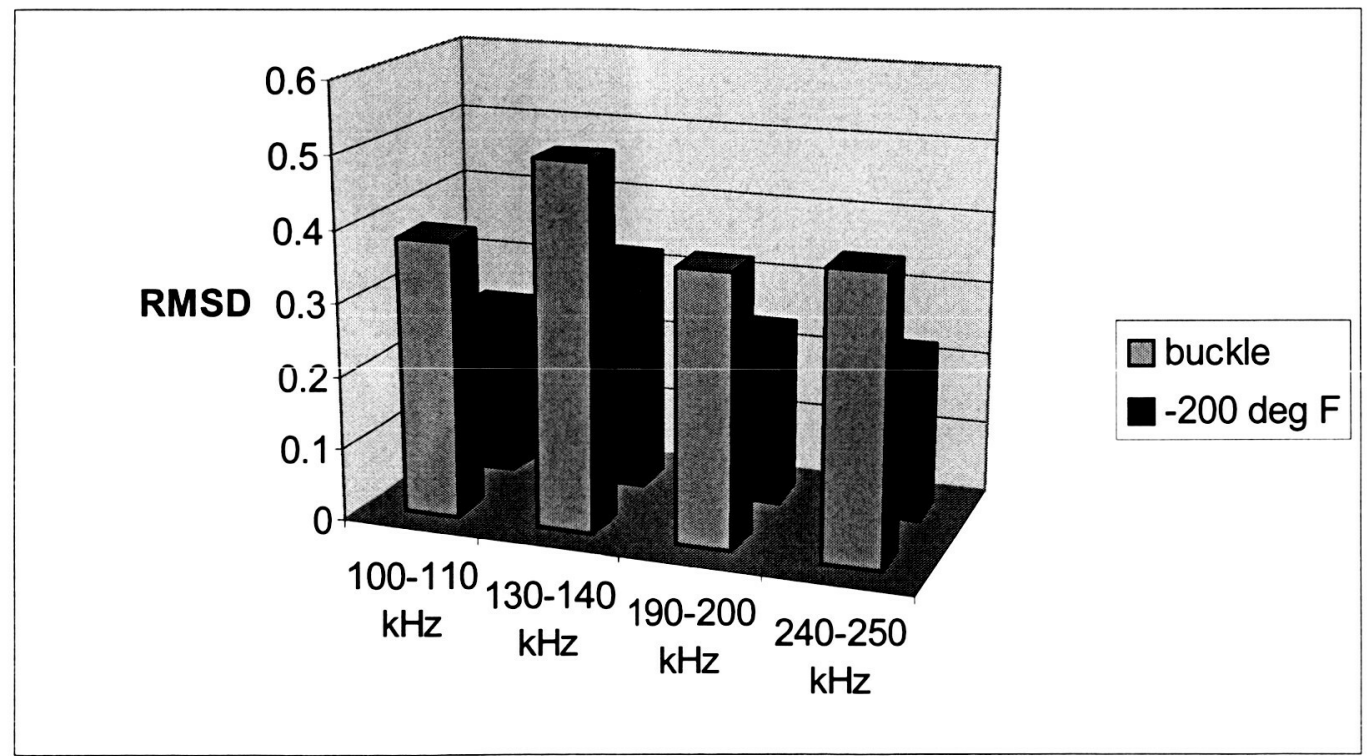

Figure 12: RMSD comparison of SCP impedance measurements of 6 x 12 panel damage to damage and low temperature. 


\section{Acknowledgments}

I would like to thank Dr. Michael Tinker of the MSFC Spacecraft Systems Analysis \& Integration Division for supervising and securing funding for much of the work presented here. Mike Kovach and other workers at ATKThiokol deserve my gratitude for skilifuiliy fabricating many of the test pieces used in this study. Speciai thanks aiso goes to Jimmy Sisco, Jerry Oakley, and other members of the MSFC Environmental and Structural Test Lab for helping provide the necessary environmental and load tests of the materials presented here.

\section{References}

${ }^{1}$ Engberg, R. and Lassiter, J. "Piezoelectric Sensor Impedance Testing Under Vacuum and Cryogenic Conditions." NASA-MSFC test report \#NGLT-DEV-03-080, January 2004.

${ }^{2}$ Liang, C., Sun, F., and Rodgers, C. "Electro-mechanical Impedance Modeling of Active Material Systems." Smart Material Structures, Vol. 51996.

${ }^{3}$ Peairs, D. Development of a Self-Sensing and Self-Healing Bolted Joint. Master's thesis, Virginia Polytechnic Institute and State University, Blacksburg, VA., July 2002.

${ }^{4}$ Roark, R. J. and W. C. Young. Formulas for Stress and Strain, $5^{\text {th }}$ ed. New York, NY: McGraw-Hill, 1975.

${ }^{5}$ Tseng, K. K. "Impedance-based Non-destructive and Non-parametric Infrastructural Performance Evaluation." Journal of Experimental Mechanics. Vol. 44 No.1, Society of Experimental Mechanics, Inc. London, UK: Sage Press, February 2004 